\title{
Nanomolar Potency Aminophenyltriazine CFTR Activator Reverses Corneal Epithelial Injury in a Mouse Model of Dry Eye
}

\author{
Xiaolan Chen,, ${ }^{1,2}$ Sujin Lee, Tianyi Zhang,, Tianying Duan,, Neel D. Pasricha, ${ }^{3}$ Julie M. Schallhorn, ${ }^{3}$ \\ Marc H. Levin, ${ }^{4}$ Vuk Koprivica, ${ }^{5}$ and Alan S. Verkman ${ }^{1}$
}

\begin{abstract}
Purpose: Dry eye disorders are a major health care burden. We previously reported the identification of $N$-methyl- $N$-phenyl-6-(2,2,3,3-tetrafluoropropoxy)-1,3,5-triazine-2,4-diamine [cystic fibrosis transmembrane conductance regulator (CFTR) act-K267], which activated human wild-type CFTR chloride conductance with $\mathrm{EC}_{50} \sim 30 \mathrm{nM}$. Here, we report in vivo evidence for $\mathrm{CFTR}_{\text {act }}-\mathrm{K} 267$ efficacy in an experimental mouse model of dry eye using a human compatible ophthalmic vehicle.

Methods: CFTR activation in mice in vivo was demonstrated by ocular surface potential difference (OSPD) measurements. Ocular surface pharmacodynamics was measured in tear fluid samples obtained at different times after topical administration of CFTR act $-\mathrm{K} 267$. Dry eye was produced by lacrimal duct cautery (LDC) and corneal epithelial injury and was assessed by Lissamine green (LG) staining.

Results: OSPD measurements demonstrated a hyperpolarization of $-8.6 \pm 3 \mathrm{mV}$ (standard error of the mean, 5 mice) in response to CFTR $_{\text {act }}-\mathrm{K} 267$ exposure in low chloride solution that was reversed by a CFTR inhibitor.

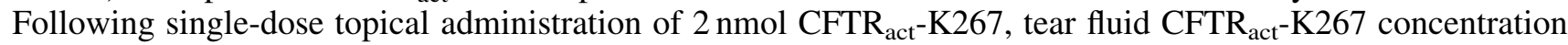
was $>500 \mathrm{nM}$ for more than $6 \mathrm{~h}$. Following LDC, corneal surface epithelial injury, as assessed by LG staining, was substantially reversed in 10 of 12 eyes receiving 2 nmol CFTR ${ }_{\text {act }}-\mathrm{K} 2673$ times daily starting on day 2 , when marked epithelial injury had already occurred. Improvement was seen in 3 of 12 vehicle-treated eyes. Conclusion: These studies provide in vivo evidence in mice for the efficacy of a topical, human use compatible CFTR $_{\text {act }}-\mathrm{K} 267$ formulation in stimulating chloride secretion and reversing corneal epithelial injury in dry eye.
\end{abstract}

Keywords: keratoconjunctivitis sicca, CFTR, chloride channel, lacrimal duct cautery

\section{Introduction}

D RY EYE DISORDERS are a major health care burden, with an estimated prevalence of 16 million people in the United States and affecting more than $10 \%$ of people older than 50 years. ${ }^{1,2}$ While heterogeneous by etiology, dry eye disease presents with common clinical signs and symptoms, which include corneal epithelial injury as assessed by fluorescein and Lissamine green (LG) staining, decreased ocular fluid and tear breakup time, and patient-reported symptoms of ocular discomfort or functional impairment in a low humidity environment. Treatment options available in the United States at present include artificial tears, punctal plugs, and the topical anti-inflammatory drugs cyclosporine and lifitegrast. ${ }^{3-6}$ Despite available options, dry eye disease is increasing in incidence and remains an unmet medical need.

We previously proposed prosecretory therapy for dry eye by targeting cystic fibrosis transmembrane conductance regulator (CFTR), a secretory chloride channel expressed in corneal and conjunctival epithelial cells at the ocular surface $^{7-9}$ As in the intestine in secretory diarrhea, activation of CFTR in cornea and conjunctiva can produce a secretory chloride current that results in net fluid secretion onto the ocular surface. Restoration of the normal aqueous layer in dry eye is predicted to reduce tear fluid hyperosmolality, a

Departments of ${ }^{1}$ Medicine and Physiology and ${ }^{3}$ Ophthalmology, University of California, San Francisco, California.

${ }^{2}$ Jiangsu Agri-Animal Husbandry Vocational College, Taizhou, China.

${ }_{5}^{4}$ Department of Ophthalmology, Palo Alto Medical Foundation, Palo Alto, California.

${ }^{5}$ Vanda Pharmaceuticals, Inc., Washington, District of Columbia. 
major driver of ocular surface inflammation and clinical symptoms, and could thus be an effective strategy to treat a broad spectrum of dry eye diseases.

We previously identified, by high-throughput screening,

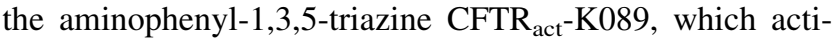
vated human wild-type CFTR with $\mathrm{EC}_{50} \sim 250 \mathrm{nM}$ and produced a secretory response at ocular surface epithelia in mice. ${ }^{10}$ Utilizing medicinal chemistry, we discovered analog $N$-methyl- $N$-phenyl-6-(2,2,3,3-tetrafluoropropoxy)1,3,5-triazine-2,4-diamine (CFTR $\left.{ }_{\text {act }}-\mathrm{K} 267\right)$, which activated CFTR chloride conductance with $\mathrm{EC}_{50} \sim 30 \mathrm{nM}$, underwent rapid hepatic metabolism (minimizing systemic exposure), and produced a sustained increase in tear volume in mice when administered topically in a dimethyl sulfoxide (DMSO)-containing ophthalmic formulation. ${ }^{11} \mathrm{CFTR}_{\mathrm{act}^{-}}$ K267 in the DMSO-containing formulation also produced a sustained secretory response in rabbits without tachyphylaxis upon 28-day repeated dosing and was detected mainly at the ocular surface without detectable blood levels. ${ }^{12}$

The purpose of this study was to test the efficacy of CFTR $_{\text {act }}$-K267, delivered in an ophthalmic vehicle suitable for use in humans, in an experimental animal model of dry eye produced by lacrimal duct cautery (LDC) in mice. The prosecretory action of $\mathrm{CFTR}_{\text {act }}-\mathrm{K} 267$ in vivo was studied by measurement of ocular surface potential differences (OSPDs), a method originally developed by our laboratory, ${ }^{9}$ and its ocular surface pharmacokinetics was measured by liquid chromatography/mass spectroscopy (LC/MS) analysis of collected tear fluid.

\section{Methods}

\section{Mice}

Female Balb/c mice (aged 7-8 weeks) were bred at the University of California, San Francisco (UCSF) Animal Facility. Animal protocols were approved by the UCSF Institutional Animal Care and Use Committee and were in compliance with the Association for Research in Vision and Ophthalmology statement for the use of animals in ophthalmic and vision research.

\section{Chemicals}

CFTR $_{\text {act }}$-K267 was synthesized by sequential substitution reactions of cyanuric chloride with $N$-methylamine, 2,2,3,3tetrafluoropropanol, and aniline under basic conditions, as described. ${ }^{11}$ CFTR $_{\text {act }}$-K267 was purified by flash column chromatography (n-hexane:ethylacetate 1:2), giving purity $>98 \%$ as determined by LC/MS. Other chemicals were purchased from Sigma-Aldrich (St. Louis, MO).

\section{Ophthalmic formulation}

CFTR $_{\text {act }}-\mathrm{K} 267(10 \mu \mathrm{mol}, 3.4 \mathrm{mg})$ was dissolved in $10 \mathrm{~mL}$ of a human compatible ophthalmic vehicle that completely dissolved the compound. The vehicle contained $50 \mathrm{mg} / \mathrm{mL}$ polyoxyl 35 castor oil, $10 \mathrm{mg} / \mathrm{mL}$ polyethylene glycol-400, $10 \mathrm{mg} / \mathrm{mL}$ propylene glycol, $2 \mathrm{mg} / \mathrm{mL} \mathrm{NaCl}$, and $2 \mathrm{mg} / \mathrm{mL}$ sodium carboxymethyl cellulose, which was brought up to a volume of $1 \mathrm{~mL}$ with $\mathrm{pH} 7.4$ phosphate buffer (prepared from $0.275 \mathrm{mg} \mathrm{NaH}{ }_{2} \mathrm{PO}_{4}$ monohydrate and $1.137 \mathrm{Na}_{2} \mathrm{PO}_{4}$ anhydrous in $1 \mathrm{~mL}$ water, titrated to $\mathrm{pH} 7.4$ with $0.1 \mathrm{M}$ phosphoric acid).

\section{OSPD measurements}

Open-circuit transepithelial potential difference (PD) in millivolts $(\mathrm{mV})$ at the ocular surface was measured in anesthetized mice as described. ${ }^{13}$ Mice were anesthetized with isoflurane, and core temperature was maintained at $37^{\circ} \mathrm{C}$ with a heating pad. The eyes were oriented with the cornea and conjunctiva facing upward. Solutions were isosmolar $(320 \pm 10 \mathrm{mOsM})$ with a $\mathrm{pH}$ of 7.4 , consisting of (in mM): solution 1: $142 \mathrm{NaCl}, 3 \mathrm{KCl}, 0.8 \mathrm{NaH}_{2} \mathrm{PO}_{4}$, $1 \mathrm{CaCl}_{2}, 0.5 \mathrm{MgCl}_{2}, 1.5 \mathrm{KH}_{2} \mathrm{PO}_{4}$; solution 2: solution $1+$ amiloride $(100 \mu \mathrm{M})$; solution 3: (low $\mathrm{Cl}^{-}$) solution 2 with $\mathrm{NaCl}$ replaced by $\mathrm{Na}$ gluconate and $\mathrm{KCl}$ by $\mathrm{K}$ gluconate; solution 4: solution $3+\mathrm{CFTR}_{\mathrm{act}}-\mathrm{K} 267(2.5$ or $10 \mu \mathrm{M})$; solution 5: solution $4+$ forskolin $(20 \mu \mathrm{M})$; solution 6: solution $5+\mathrm{CFTR}_{\mathrm{inh}}-172(10 \mu \mathrm{M})$. The ocular surface was perfused at $6 \mathrm{~mL} / \mathrm{min}$ through plastic tubing with a multireservoir gravity pinch-valve system and variable-flow peristaltic pump. A probe catheter was immobilized $1 \mathrm{~mm}$ above the cornea with a micropositioner, and a suction cannula was positioned $3 \mathrm{~mm}$ from the orbit. The measuring electrode contacting the perfusion catheter was connected to a highimpedance voltmeter (IsoMilivolt Meter; World Precision Instruments), with reference consisting of a 21-gauge needle filled with isosmolar saline inserted subcutaneously. Measuring and reference electrodes consisted of $\mathrm{Ag} / \mathrm{AgCl}$ with $3 \mathrm{M} \mathrm{KCl}$ agar bridges.

\section{Ocular pharmacokinetics}

Mice were topically given $2 \mu \mathrm{L}$ of $1 \mathrm{mM} \mathrm{CFTR}_{\text {act }}-\mathrm{K} 267$ in ophthalmic vehicle by micropipette. At specified times, 3 eye washes [ $3 \mu \mathrm{L}$ phosphate-buffered saline (PBS) each] were recovered from the lateral and medial canthi using $5 \mu \mathrm{L}$ microcapillary tubes (Drummond Scientific Co., Broomhall, PA) after manual eyelid blinking. $6 \mu \mathrm{L}$ of pooled washes were mixed with $34 \mu \mathrm{L}$ of eluent solution (1:1 mixture of acetonitrile/water containing $0.1 \%$ formic acid) and analyzed by LC/MS. LC was performed on a Xterra MS C18 column $(2.1 \times 100 \mathrm{~mm}, 3.5 \mu \mathrm{m})$ with $0.2 \mathrm{~mL} / \mathrm{min}$ water/acetonitrile containing $0.1 \%$ formic acid, 24-min linear gradient, and $5 \%$ to $95 \%$ acetonitrile connected to a 2695 HPLC (Waters, Milford, MA) solvent delivery system. Low-resolution MS was performed on a Micromass ZQ mass spectrometer (Waters) with positive electrospray ionization.

\section{LDC model}

A LDC model was used as described, ${ }^{10}$ modified from Stevenson et al. ${ }^{13}$ On day 0 , the extraorbital lacrimal glands were exposed bilaterally by $3-\mathrm{mm}$ linear skin incisions. The linear duct was reliably visualized along its course to the lateral canthus by gently lifting the main lacrimal gland from the face with blunt forceps. The lacrimal duct on each side was ablated with a high-temperature handheld cautery pen, avoiding facial vessels and nerves. Incisions were closed with a single, interrupted 6-0 silk suture. Accessory lacrimal tissue remained intact. On day 2 (and in some studies on day 0 just before LDC), to assess corneal epithelial integrity, $5 \mu \mathrm{L}$ of $1 \% \mathrm{LG}$ dye was applied to the ocular surface of isoflurane-anesthetized mice and washed after $10 \mathrm{~s}$ with PBS. Two photographs of each eye were taken with a digital camera (Canon, Revel T5i, Japan) adapted to a zoom stereo microscope (Nikon, SMZ1500, Japan). After 
LG staining on day 2, mouse eyes were randomized to receive CFTR $_{\text {act }}-\mathrm{K} 267$ treatment in one eye and vehicle in the other eye. Treatment was 2 nmol CFTR $_{\text {act }}-\mathrm{K} 267$ in $2 \mu \mathrm{L}$ ophthalmic vehicle given 3 times per day (at 9 AM, 2 PM, and 7 PM) for 5 days starting on day 2. LG staining was also performed on days 4 and 6 at $2 \mathrm{~h}$ after the 7 PM eye drop.

\section{Data analysis and statistics}

Photographs from LG staining were scored by 2 investigators who were unaware of treatment status, with each grader using a different scoring method. In the first scoring method, each corneal quadrant was scored on a 3-point scale with staining in each quadrant classified as: grade 0 , no staining; grade 1, sporadic staining (involving $<25 \%$ of the total surface); grade 2, diffuse punctate staining (25\%$75 \%)$; grade 3 , coalesced punctate staining $(\geq 75 \%)$. The total "Lissamine green score" was determined as the sum of scores from all four quadrants, ranging from 0 to 12 . In the second scoring method giving a "Comparative Lissamine green grade," photos from days 2, 4, and 6 were scored comparatively, comparing $\mathrm{R}$ versus $\mathrm{L}$ eyes in each mouse $(0$, no difference; $+1, \mathrm{R}$ eye with mildly more staining than L eye; +2 , $\mathrm{R}$ eye with much more staining than left eye; -1 and $-2, \mathrm{R}$ eye with mildly and much less staining than $\mathrm{L}$ eye). Data are presented as mean \pm standard error of the mean (SEM). Statistical analyses were performed using GraphPad prism 8 software with statistical comparisons made using the paired 2-tailed $t$-test.

\section{Results}

\section{OSPD measurement}

CFTR $_{\text {act }}-\mathrm{K} 267$ activation of CFTR chloride conductance at the ocular surface was studied in live mice using an ocular surface PD measurement method in which the PD (in $\mathrm{mV}$ ) generated by the ocular surface epithelium in situ was measured using a high-impedance voltmeter with sensing electrodes in electrical contact with the ocular surface and body (Fig. 1A). Initial ocular surface PD measured using a physiological chloride-containing solution in the perfusate (solution 1, see the Methods section) was $-30 \pm 8 \mathrm{mV}$ (SEM, $n=5$ eyes), which depolarized by $3.4 \pm 1.8 \mathrm{mV}$ following addition of amiloride (solution 2) to inhibit the epithelial sodium channel $(\mathrm{ENaC})$. A representative curve is shown at the left and data from separate experiments along with averaged values for PD changes $(\Delta \mathrm{PD})$ are shown on the right. Then, perfusion with a low chloride solution containing amiloride (solution 3) produced a hyperpolarization in part due to a diffusion potential. Perfusion with CFTR $_{\text {act }}-\mathrm{K} 267$ at $10 \mu \mathrm{M}$ (solution 4), followed by the CFTR agonist forskolin (solution 5) produced hyperpolarizations of $8.6 \pm 2.7 \mathrm{mV}$ and $7.8 \pm 4.5 \mathrm{mV}$, respectively. Perfusion with solution contain-

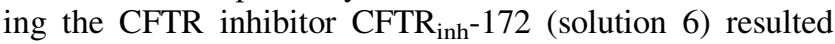
in a depolarization of $7.3 \pm 4.5 \mathrm{mV}$. Figure $1 \mathrm{C}$ shows a similar study but with $2.5 \mu \mathrm{M} \mathrm{CFTR}_{\text {act }}-\mathrm{K} 267$, a concentration chosen from pilot studies as producing a robust response. A slow $3.0 \pm 1.0 \mathrm{mV}$ hyperpolarization was seen with $2.5 \mu \mathrm{M}$ $\mathrm{CFTR}_{\mathrm{act}}-\mathrm{K} 267$ in the low chloride solution. The results
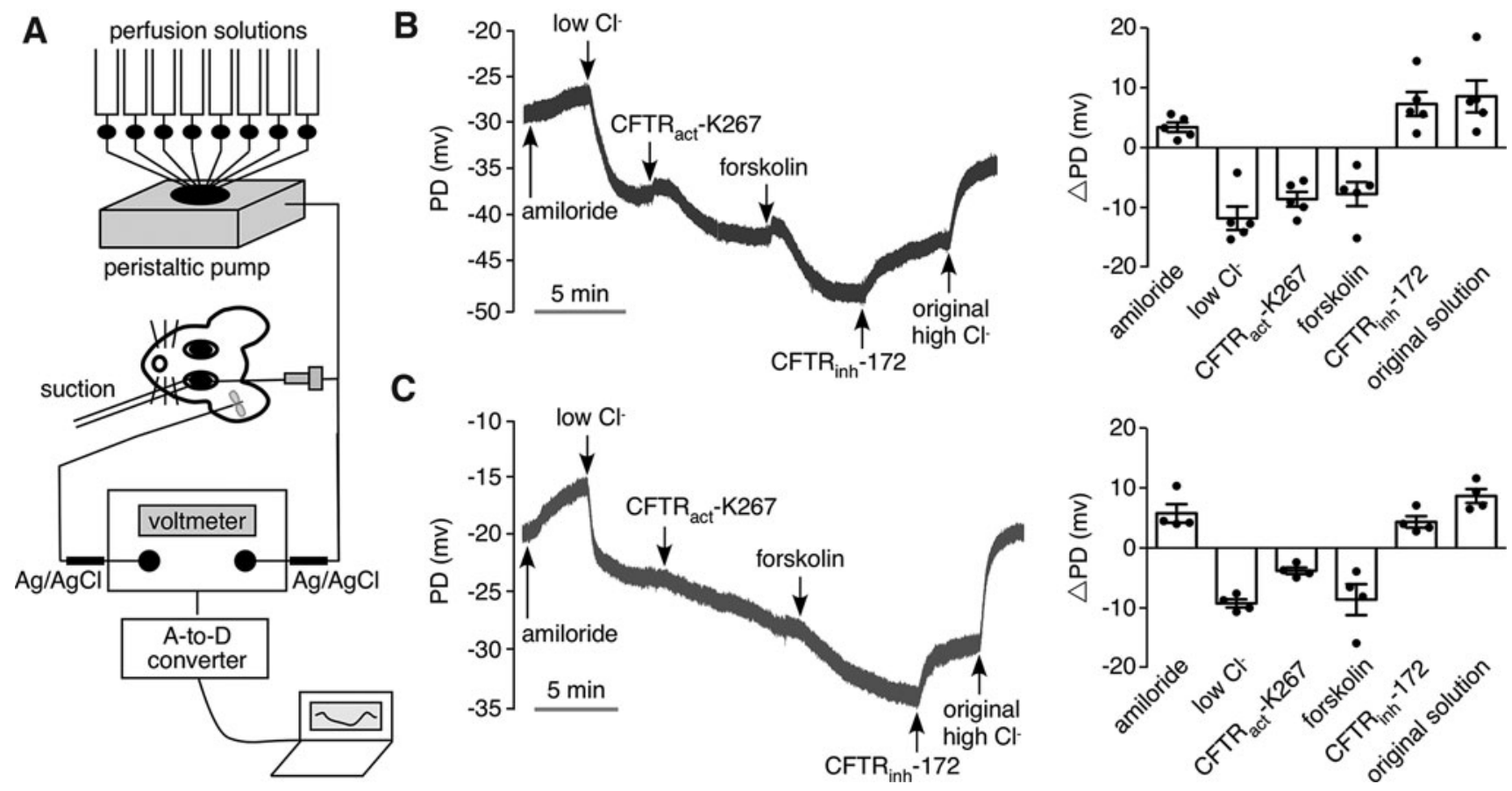

FIG. 1. CFTR act $^{-K 267}$ activation of CFTR chloride conductance at the ocular surface in mice studied by ocular surface PD measurements. (A) Schematic of the method showing perfusion of the ocular surface with different solutions and measurement of PD using a high-impedance voltmeter, with sensing electrode in electrical contact with the perfusion solution and grounding needle electrode placed under the skin. (B, C) (Left) Representative continuous recordings of ocular surface $\mathrm{PD}$ as a function of time in response to perfusion with solutions containing indicated compounds (see the Methods section for solution compositions). Concentrations of $\mathrm{CFTR}_{\text {act }}-\mathrm{K} 267$ were $10 \mu \mathrm{M}(\mathbf{B})$ and $2.5 \mu \mathrm{M}(\mathbf{C})$. (Right) Summary of changes in ocular surface PD $(\triangle \mathrm{PD})$ for successive solution changes. Each point is from a study performed on a different eye (mean $\pm \mathrm{SEM}$ ). CFTR, cystic fibrosis transmembrane conductance regulator; PD, potential difference; SEM, standard error of the mean. 

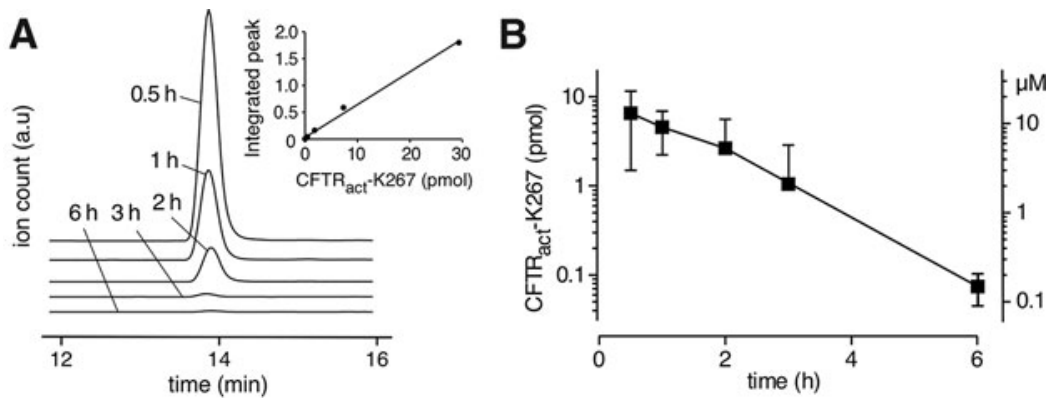

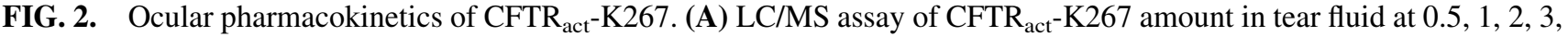
and $6 \mathrm{~h}$ after single-dose $(2 \mathrm{nmol})$ administration as $2 \mu \mathrm{L}$ eye drop. Representative chromatograms $(\mathrm{m} / \mathrm{z}=332)$ shown, with standard curve shown as inset. (B) Averaged recovered CFTR act $^{-K 267}$ (left ordinate) and deduced CFTR act $^{-K_{2}} 267$ concentration (right ordinate) computed assuming a $0.5 \mu \mathrm{L}$ tear fluid volume per eye (mean \pm SEM, 4 eyes per time point). LC/MS, liquid chromatography/mass spectroscopy.

provide in vivo evidence for $\mathrm{CFTR}_{\text {act }}-\mathrm{K} 267$ activation of CFTR chloride conductance at the ocular surface.

\section{Tear fluid pharmacokinetics}

The amount of CFTR $_{\text {act }}-\mathrm{K} 267$ in tear fluid at different times after single-dose topical administration of $2 \mathrm{nmol}$ CFTR $_{\text {act }}$-K267 was determined by LC/MS analysis of combined fluid from 3 eye washes, using different mice for each time point. Figure 2A (inset) shows an approximately linear standard curve for LC/MS integrated peak ion current as a function of $\mathrm{CFTR}_{\text {act }}-\mathrm{K} 267$ amount from which $\mathrm{CFTR}_{\text {act }}-\mathrm{K} 267$ in the combined eye washes was determined. Figure 2A also shows original LC/MS curves for recovered CFTR $_{\text {act }}-\mathrm{K} 267$ per eye at different times after eye drop administration. Averaged results from 4 eyes per time point showed an approximately exponential reduction in $\mathrm{CFTR}_{\mathrm{act}^{-}}$ K267 amount with time, with computed $t_{1 / 2}$ of $62 \mathrm{~min}$ (Fig. 2B). The deduced tear film concentration of CFTR act $^{-}$ $\mathrm{K} 267$, computed assuming a tear fluid volume of $0.5 \mu \mathrm{L}$ per eye, ${ }^{14}$ is shown on the right-hand ordinate. The estimated $\mathrm{CFTR}_{\text {act }}-\mathrm{K} 267$ concentration was well above its $\mathrm{EC}_{50}$ of $\sim 30 \mathrm{nM}$, as measured in cell studies, ${ }^{11}$ for at least $6 \mathrm{~h}$ after single-dose administration. The amounts and deduced concentrations of $\mathrm{CFTR}_{\mathrm{act}}-\mathrm{K} 267$ may underestimate the actual values by $15 \%-20 \%$ based on the recovery efficiency of $80 \%-85 \%$ estimated previously using a fluorescent fluidphase marker. $^{10}$

\section{LDC model of dry eye}

A robust $\mathrm{LDC}$ model was implemented in mice in which cautery of the main lacrimal duct, located between the inferior ear lobe and the lateral canthus, resulted in consistent corneal epithelial injury as determined by LG staining (Fig. 3A). Representative photographs of mouse corneas show little LG staining before LDC, with marked staining of the same ocular surfaces at 2 days after LDC (Fig. 3B). No increase in LG staining was seen in sham-operated eyes in which the cautery step was omitted (data not shown). Figure 3C summarizes corneal epithelial injury scores before and 2 days after LDC in 12 eyes studied. All operated eyes show moderate epithelial disruption within a narrow range.

To study the efficacy of $\mathrm{CFTR}_{\mathrm{act}}-\mathrm{K} 267$, delivered using an optimized ophthalmic vehicle, in reversing corneal epithelial injury, the formulation studied in Fig. 2 was administered to mice 3 times daily beginning after the LG staining on day 2, with LG staining repeated on days 4 and 6 (Fig. 4A). For each mouse, 1 eye was administered $2 \mu \mathrm{L}$ of CFTR $_{\text {act }}-$ K267-containing formulation $(2 \mathrm{nmol})$ and the
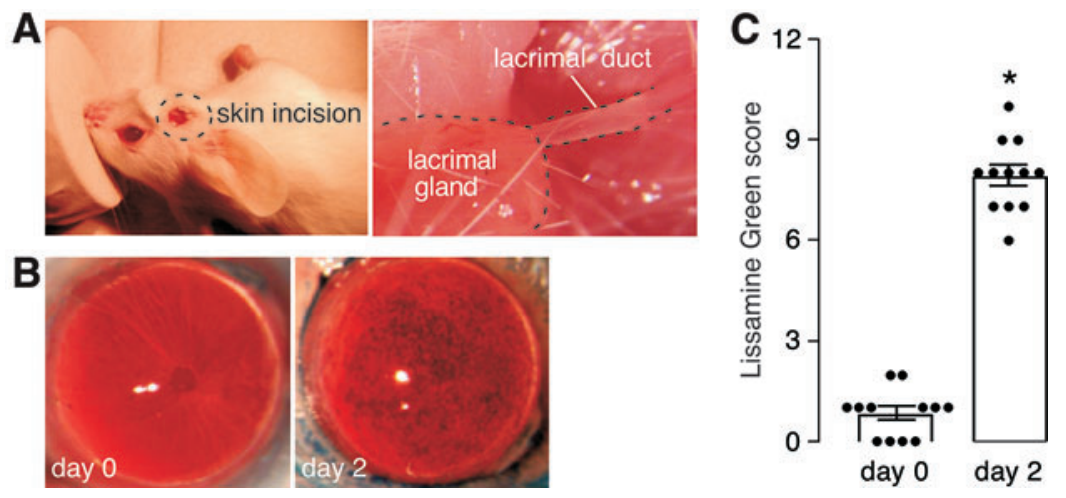

FIG. 3. LDC model of dry eye disease in mice. (A) LDC was produced by high-temperature cautery of the main lacrimal duct bilaterally, identified following a short linear skin excision to expose the main lacrimal gland and its duct. (B) Representative photos of LG staining of cornea just before and 2 days after bilateral LDC. (C) Scoring of corneal epithelial injury deduced from photographs as in (B) using a 12-point standard scale (see the Methods section) (12 eyes, mean \pm SEM, $* P<0.001)$. LDC, lacrimal duct cautery; LG, Lissamine green. Color images are available online. 

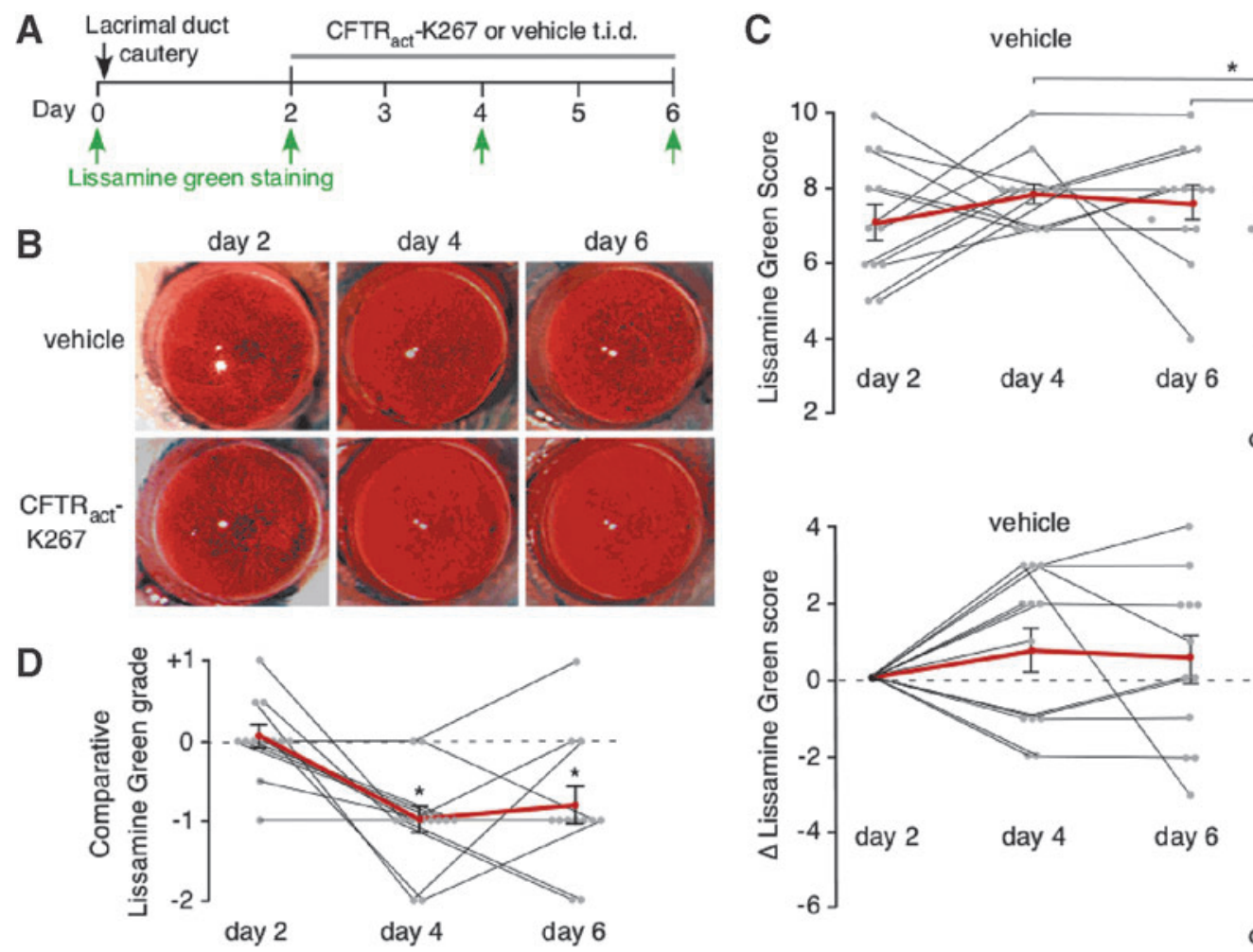

FIG. 4. Topical $\mathrm{CFTR}_{\mathrm{act}}-\mathrm{K} 267$ reverses corneal epithelial injury in mice following LDC. (A) Experimental protocol. (B) Representative photos of LG staining of cornea at days 2, 4, and 6 after LDC in vehicle and CFTR act $^{-K_{2}}$ 267-treated eyes. (C) Photos scored using a 12-point standard scale (see the Methods section). (Top) Absolute LG scores on day 2 (2 days after

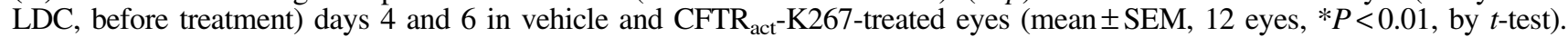
(Bottom) Data shown for each individual eye (black lines) as the change in LG scores from those on day 2. Averaged data are shown as red lines (mean \pm SEM, 12 eyes, $* P<0.05$ compared with day 2 , by $t$-test). (D) Photos scored using a comparative grading method (see the Methods section). Data shown for individual mice as Comparative LG grade for CFTR act $^{-K 267-}$ versus vehicle-treated eyes (mean \pm SEM, 12 eyes, $* P<0.01$ compared with day 2 , by $t$-test). Color images are available online.

other eye was administered control vehicle, with the treated eye (left vs. right) randomized. A total of 12 mice ( 6 per group) were studied. Representative LG photographs of CFTR $_{\text {act }}-$ K267-treated and contralateral vehicle-treated eyes are shown in Fig. 4B.

LG photographs of corneas were independently scored by 2 investigators (M.H.L. and A.S.V.) who were unaware of eye treatment status and using different scoring systems. Utilizing a standard 12-point scoring system that summed staining in each quadrant of each cornea, ${ }^{15}$ there was a significant reduction in staining score in the $\mathrm{CFTR}_{\text {act }}-\mathrm{K} 267-$ treated eyes at days 4 and 6, shown as absolute staining scores (Fig. 4C, top) or as the difference $(\Delta)$ in staining score (Fig. 4C, bottom). No significant change in staining score was seen in the vehicle-treated eyes. Improvement in score was seen in 10 of 12 of the $\mathrm{CFTR}_{\text {act }}-\mathrm{K} 267$ treated eyes. Using an independent, comparative grading system in which LG staining in left and right eyes was compared in each mouse, there was a significant improvement in the "Comparative Lissamine green score" on days 4 and 6, with improvement seen in 10 of 12 CFTR $_{\text {act }}-K_{2} 67$-treated eyes on day 4 and in 9 of 12 on day 6 (Fig. 4D).

\section{Discussion}

The data herein provide evidence for the efficacy of aminophenyltriazine CFTR $_{\text {act }}-\mathrm{K} 267$ in activating CFTR chloride conductance at the ocular surface in mice and in reversing corneal epithelial injury in a surgical mouse model of aqueous-deficient dry eye. For these studies, a DMSOfree ophthalmic formulation was established for stable dissolution and topical ocular delivery of CFTR $_{\text {act }}-\mathrm{K} 267$, which by ocular pharmacokinetics measurements produced predicted therapeutic levels of CFTR $_{\text {act }}-\mathrm{K} 267$ in the tear film for at least $6 \mathrm{~h}$ following single-dose administration. CFTR $_{\text {act }}-\mathrm{K} 267$ efficacy in producing a CFTR-mediated chloride secretory response at the ocular surface in mice was demonstrated by open-circuit PD measurements in which the addition of $\mathrm{CFTR}_{\mathrm{act}}-\mathrm{K} 267$ in a low chloride-containing solution resulted in a hyperpolarization of magnitude comparable to that produced by the cAMP agonist forskolin. A mouse model of dry eye involving LDC showed consistent and robust corneal epithelial injury 2 days after cautery, which was largely reversed by topical CFTR $_{\text {act }}-\mathrm{K}_{2} 67$.

The corneal epithelium transports chloride from the basal side to the tear side to maintain corneal hydration and clarity. ${ }^{16}$ Expression of several chloride transporters has been reported in corneal epithelial cells, including CLCA2, a putative calcium-activated chloride channel seen in human and chick corneal epithelial cells, ${ }^{17}$ and CFTR in mouse, rabbit, and human cornea. ${ }^{10,12,18}$ In the eye, CFTR is also expressed in corneal endothelium and retinal pigment epithelium, where it has been proposed to facilitate corneal deturgescence $^{19}$ and regulate subretinal extracellular volume 
and ionic content. ${ }^{20,21}$ CFTR has also been localized to apical superficial cell membranes of the stratified conjunctival epithelium ${ }^{22,23}$ and in lacrimal gland epithelium, ${ }^{7}$ suggesting a role in tear film homeostasis and as a target for therapy of dry eye.

A prosecretory therapeutic is anticipated to be beneficial in dry eye disease from a variety of etiologies such as aqueous deficiency, as seen in Sjögren's syndrome, but also in evaporative dry eye states. ${ }^{24,25}$ Increasing tear fluid volume on the ocular surface would reduce tear hyperosmolality and the downstream inflammatory response. Currently, 5\% lifitegrast ophthalmic solution is Food and Drug Administration approved to treat dry eye in the United States. $0.05 \%$ cyclosporine ophthalmic emulsion, which is also routinely used to treat dry eye, is formally approved to normalize tear secretion, although by an uncertain mechanism. These are both anti-inflammatory drugs that limit the ocular surface inflammatory response common in dry eye patients. In Japan, 3\% diquafosol ophthalmic solution, which stimulates a prosecretory response through the $\mathrm{P} 2 \mathrm{Y} 2$ purinergic receptor, and $2 \%$ rebamipide ophthalmic suspension, which has a stabilizing effect on the ocular surface mucin layer, have been approved. ${ }^{26}$ Other therapeutic approaches for addressing dry eye are being investigated. ${ }^{27}$

Our original studies were performed with a vehicle containing DMSO, ${ }^{11}$ which can solubilize a wide variety of otherwise poorly soluble polar and nonpolar molecules. With the goal of translating a prosecretory CFTR-activating compound to human clinical trials, a non-DMSO-containing formulation, incorporating ocular dosing-approved vehicles, was evaluated here. $\mathrm{CFTR}_{\mathrm{act}}-\mathrm{K} 267$ was stably dissolved in the formulation, which when delivered topically to mice showed favorable pharmacokinetics and efficacy in reversing dry eye. The human compatible $\mathrm{CFTR}_{\mathrm{act}}-\mathrm{K} 267$ formulation reversed the effects of lacrimal gland cautery in the majority of treated eyes following 2 days of treatment, and a single dose gave ocular fluid concentrations that are predicted to be sufficient for CFTR activation over at least $6 \mathrm{~h}$. The data suggest that, if it passes safety testing, CFTR act $^{-}$ K267 could be a potential treatment for dry eye associated with decreased ocular tear film. These results further support that potential utility of stimulating CFTR secretory activity on the ocular surface for the treatment of dry eye disease.

In summary, CFTR act $^{-K 267}$ in a human use compatible ophthalmic formulation increased chloride secretion at the ocular surface and reversed corneal epithelial injury in an experimental mouse model of dry eye. Ocular fluid pharmacokinetics studies showed predicted therapeutic concentrations of $\mathrm{CFTR}_{\mathrm{act}}-\mathrm{K} 267$ for at least $6 \mathrm{~h}$ following single-dose topical administration, supporting further development of $\mathrm{CFTR}_{\mathrm{act}}{ }^{-}$ K267 as a candidate dry eye therapeutic.

\section{Acknowledgments}

The authors acknowledge Drs. Udaya Kotreka, Rakesh Koutlekar, and Shawn Dressman from Vanda Pharmaceuticals for formulation and CMC contributions.

\section{Authors' Contributions}

M.H.L., V.K., and A.S.V. designed experiments. T.Z. and T.D. performed potential difference measurements. X.C. and S.L. performed pharmacokinetic studies. X.C. performed lacrimal duct ligation studies. M.H.L. and A.S.V. analyzed lacrimal gland ligation studies. All authors contributed to writing of the article.

\section{Author Disclosure Statement}

S.L., M.H.L., and A.S.V. are named inventors on patent applications on CFTR activators for dry eye owned by the University of California, San Francisco. M.H.L. and A.S.V. are consultants for Vanda Pharmaceuticals, Inc., and V.K. is an employee of Vanda Pharmaceutics, Inc. The CFTR act $^{-}$ K267 patents are licensed to Vanda Pharmaceutics, Inc. The other authors have nothing to disclose.

\section{Funding Information}

This work was supported by grants EY13574, DK72517, and DK101373 from the National Institutes of Health and a grant from the Cystic Fibrosis Foundation, and sponsored research from Vanda Pharmaceuticals, Inc. X.C. was supported by a 2018 grant program from Qinglan Engineering of Jiangsu Province.

\section{References}

1. Dana, R., Bradley, J.L., Guerin, A., et al. Estimated prevalence and incidence of dry eye disease based on coding analysis of a large, all-age United States Health Care System. Am. J. Ophthalmol. 202:47-54, 2019.

2. Bradley, J.L., Ozer Stillman, I., Pivneva, I., et al. Dry eye disease ranking among common reasons for seeking eye care in a large US claims database. Clin. Ophthalmol. 13: 225-232, 2019.

3. Kuklinski, E., and Asbell, P.A. Sjogren's syndrome from the perspective of ophthalmology. Clin. Immunol. 182:5561, 2017.

4. Pucker, A.D., Ng, S.M., and Nichols, J.J. Over the counter (OTC) artificial tear drops for dry eye syndrome. Cochrane Database Syst. Rev. 2:CD009729, 2016.

5. Song, J.S., Woo, I.H., Eom, Y., and Kim, H.M. Five misconceptions related to punctal plugs in dry eye management. Cornea 37 Suppl 1:S58-S61, 2018.

6. Yang, J.M., Choi, W., Kim, N., and Yoon, K.C. Comparison of topical cyclosporine and diquafosol treatment in dry eye. Optom. Vis. Sci. 92:e296-e302, 2015.

7. Berczeli, O., Vizvari, E., Katona, M., et al. Novel insight into the role of CFTR in lacrimal gland duct function in mice. Invest. Ophthalmol. Vis. Sci. 59:54-62, 2018.

8. Nandoskar, P., Wang, Y., Wei, R., et al. Changes of chloride channels in the lacrimal glands of a rabbit model of Sjogren syndrome. Cornea 31:273-279, 2012.

9. Levin, M.H., and Verkman, A.S. CFTR-regulated chloride transport at the ocular surface in living mice measured by potential differences. Invest. Ophthalmol. Vis. Sci. 46: 1428-1434, 2005.

10. Flores, A.M., Casey, S.D., Felix, C.M., Phuan, P.W., Verkman, A.S., and Levin, M.H. Small-molecule CFTR activators increase tear secretion and prevent experimental dry eye disease. FASEB J. 30:1789-1797, 2016.

11. Lee, S., Phuan, P.W., Felix, C.M., Tan, J.A., Levin, M.H., and Verkman, A.S. Nanomolar-potency aminophenyl1,3,5-triazine activators of the cystic fibrosis transmembrane conductance regulator (CFTR) chloride channel for prosecretory therapy of dry eye diseases. J. Med. Chem. 60: 1210-1218, 2017. 
12. Felix, C.M., Lee, S., Levin, M.H., and Verkman, A.S. Prosecretory activity and pharmacology in rabbits of an aminophenyl-1,3,5-triazine CFTR activator for dry eye disorders. Invest. Ophthalmol. Vis. Sci. 58:4506-4513, 2017.

13. Stevenson, W., Chen, Y., Lee, S.M., et al. Extraorbital lacrimal gland excision: a reproducible model of severe aqueous tear-deficient dry eye disease. Cornea 33:13361341, 2014.

14. Sullivan, D.A., Krenzer, K.L., Sullivan, B.D., Tolls, D.B., Toda, I., and Dana, M.R. Does androgen insufficiency cause lacrimal gland inflammation and aqueous tear deficiency? Invest. Ophthalmol. Vis. Sci. 40:1261-1265, 1999.

15. Vijmasi, T., Chen, F.Y., Chen, Y.T., Gallup, M., and McNamara, N. Topical administration of interleukin-1 receptor antagonist as a therapy for aqueous-deficient dry eye in autoimmune disease. Mol. Vis. 19:1957-1965, 2013.

16. Cao, L., Zhang, X.D., Liu, X., Chen, T.Y., and Zhao, M. Chloride channels and transporters in human corneal epithelium. Exp. Eye Res. 90:771-779, 2010.

17. Connon, C.J., Kawasaki, S., Liles, M., et al. Gene expression and immunolocalisation of a calcium-activated chloride channel during the stratification of cultivated and developing corneal epithelium. Cell Tissue Res. 323:177182, 2006.

18. Fan, T., Zhao, J., Ma, X., Xu, X., Zhao, W., and Xu, B. Establishment of a continuous untransfected human corneal endothelial cell line and its biocompatibility to denuded amniotic membrane. Mol. Vis. 17:469-480, 2011.

19. Sun, X.C., and Bonanno, J.A. Expression, localization, and functional evaluation of CFTR in bovine corneal endothelial cells. Am. J. Physiol. Cell Physiol. 282:C673-C683, 2002.

20. Reigada, D., and Mitchell, C.H. Release of ATP from retinal pigment epithelial cells involves both CFTR and vesicular transport. Am. J. Physiol. Cell Physiol. 288: C132-C140, 2005.

21. Blaug, S., Quinn, R., Quong, J., Jalickee, S., and Miller, S.S. Retinal pigment epithelial function: a role for CFTR? Doc. Ophthalmol. 106:43-50, 2003.
22. Turner, H.C., Bernstein, A., and Candia, O.A. Presence of CFTR in the conjunctival epithelium. Curr. Eye Res. 24: 182-187, 2002.

23. Shiue, M.H., Gukasyan, H.J., Kim, K.J., Loo, D.D., and Lee, V.H. Characterization of cyclic AMP-regulated chloride conductance in the pigmented rabbit conjunctival epithelial cells. Can. J. Physiol. Pharmacol. 80:533-540, 2002.

24. Jones, L., Downie, L.E., Korb, D., et al. TFOS DEWS II management and therapy report. Ocul. Surf. 15:575-628, 2017.

25. Craig, J.P., Nichols, K.K., Akpek, E.K., et al. TFOS DEWS II definition and classification report. Ocul. Surf. 15:276283, 2017.

26. Koh, S. Clinical utility of $3 \%$ diquafosol ophthalmic solution in the treatment of dry eyes. Clin. Ophthalmol. 9: 865-872, 2015.

27. Markoulli, M., and Hui, A. Emerging targets of inflammation and tear secretion in dry eye disease. Drug Discov. Today 24:1427-1432, 2019.

Received: August 14, 2019

Accepted: December 5, 2019

Address correspondence to: Dr. Alan S. Verkman

1246 Health Sciences East Tower University of California, San Francisco 513 Parnassus Avenue San Francisco, CA 94143-0521

E-mail: alan.verkman@ucsf.edu

Dr. Vuk Koprivica

Vanda Pharmaceuticals, Inc. 2200 Pennsylvania Avenue Northwest, Suite 300 E Washington, DC 20037

E-mail: vuk.koprivica@vandapharma.com 